\section{Desigualdades socioeconômicas e demográficas como fatores de risco para a artrite autorreferida: estudo de base populacional em adultos no Sul do Brasil}

\author{
Socioeconomic and demographic inequalities as risk \\ factors for self-reported arthritis: a population- \\ based study in southern Brazil
}

1 Programa de Pós-graduação
em Saúde Coletiva,
Universidade Federal
de Santa Catarina,
Florianópolis.
Correspondência
R. S. Gomes
Programa de Pós-graduação
em Saúde Coletiva,
Universidade Federal de
Santa Catarina.
Campus Universitário
Trindade, Florianópolis, SC
88010-970, Brasil.
gomesmed2002@ibest.com.br

\section{Abstract}

The study aimed to estimate prevalence of selfreported arthritis or rheumatism and associated factors. This was a cross-sectional populationbased study in Florianopolis, Santa Catarina State, Brazil, with 1,720 adults ranging from 20 to 59 years of age. Presence of self-reported arthritis or rheumatism was analyzed with a hierarchical approach, considering demographic, socioeconomic, and behavioral variables and use of health services. Logistic regression was used to evaluate the association between the outcome and independent variables. Prevalence of self-reported arthritis or rheumatism was 7.7\% (95\%CI: 6.4-8.9). The odds of self-reported arthritis were twice as high in women, and increased self-reported arthritis was directly associated with BMI $\geq 30 \mathrm{~kg} / \mathrm{m}^{2}$ and increasing age and inversely proportional to schooling. Self-reported arthritis or rheumatism was higher in this sample than in Brazilian adults in general in 2008. The results suggest the need to plan public health policies to address this problem.

Arthritis; Rheumatic Diseases; Adult; Prevalence
Rafael Santos Gomes 1

Karen Glazer Peres 1

\section{Introdução}

A Organização Mundial da Saúde (OMS) denominou o período de 2000-2010 como a "década do osso e da articulação" por conta do aumento na prevalência das doenças reumáticas e consequente impacto socioeconômico desencadeado por esse agravo ${ }^{1}$. Os termos artrite ou reumatismo são utilizados no senso comum para determinar uma série de doenças reumatológicas que comprometem o sistema músculo-esquelético 2 . A osteoartrite ou artrose, doença degenerativa articular, de caráter crônico, é o tipo mais comum de artrite, correspondente a cerca de $70 \%$ dos casos 3,4 .

Estima-se que a prevalência de artrite autorreferida ou diagnosticada pelo médico nos Estados Unidos aumente de $28,8 \%$ para $32,2 \%$ do ano de 2005 para 2050 em adultos de 20 anos ou mais de idade, representando, em termos absolutos, um aumento de 60 para 96 milhões de pessoas acometidas 5 . No ano de 2002, 4,8\% dos adultos norte-americanos entre 18 e 64 anos apresentaram limitação na atividade laboral atribuída à artrite, tornando-se a terceira causa mais comum de afastamento das atividades de trabalho, ficando atrás somente das doenças da coluna e das doenças do coração 6,7. Um inquérito em saúde, de base populacional, realizado no Estado de Victoria, Austrália, mostrou que $23 \%$ dos adultos reportavam ter artrite 8 . Apesar da incapacidade por artrite ou reumatismo ser mais provável no 
idoso, um estudo realizado no Reino Unido estimou essa condição em 82 de cada mil indivíduos a partir dos 16 anos de idade ${ }^{9}$.

No Brasil, inquéritos epidemiológicos de saúde, como a Pesquisa Nacional por Amostra de Domicílios (PNAD), também apontam dados sobre a artrite 10,11. A PNAD de 1998 revelou que a artrite ou reumatismo foi a terceira condição de saúde autorreferida mais prevalente na população geral, sendo mais comum entre as mulheres $(10,4 \%)$ do que entre os homens $(5,8 \%)$, ficando somente atrás da doença da coluna ou das costas e hipertensão arterial sistêmica, respectivamente 11 .

Já nos anos de 2003 e 2008, os resultados da PNAD demonstraram que a prevalência de artrite ou reumatismo foi de $6,1 \%$ e $5,7 \%$ na população de adultos com 18 anos ou mais, respectivamente, mantendo a terceira posição das doenças crônicas autorreferidas 10 .

As informações disponíveis sobre tal agravo no contexto brasileiro têm origem, em sua maioria, em pesquisas de âmbito nacional ou em municípios de pequeno porte $4,12,13$, sendo escassos estudos de base populacional sobre artrite realizados em municípios de porte maior e nas capitais brasileiras. A busca bibliográfica sobre prevalência autorreferida de artrite ou reumatismo para população adulta brasileira realizada nas bases de dados MEDLINE, LILACS e SciELO de 1991 até março de 2011 encontrou oito artigos publicados sobre o assunto, apesar do grande impacto das doenças reumáticas no sistema de saúde dos países desenvolvidos e mais recentemente do Brasil. Por exemplo, 123.929 atendimentos ambulatoriais foram realizados pelo Sistema Único de Saúde (SUS) na especialidade de Reumatologia, no Município de São Paulo em 2004 14. Adicionalmente, 36.042 atendimentos reumatológicos ocorreram no Distrito Federal no ano de 2007, estimando-se uma demanda reprimida de 16.664 usuários do SUS 15.

Identificar fatores associados à artrite pode fornecer subsídios para planejar e organizar programas específicos de assistência à saúde na atenção primária para rastreamento, prevenção e tratamento precoce e, consequentemente, evitar a incapacidade e melhorar a qualidade de vida da população acometida pela doença 4,8,16.

O presente estudo teve como objetivo estimar a prevalência e identificar os fatores socioeconômicos, demográficos e de comportamentos relacionados à saúde, associados ao diagnóstico autorreferido de artrite ou reumatismo em adultos de Florianópolis, Santa Catarina, Brasil, no ano de 2009.

\section{Métodos}

Trata-se de um estudo seccional, de base populacional, realizado entre setembro de 2009 e janeiro de 2010 com adultos de 20-59 anos, de ambos os sexos, residentes na zona urbana do Município de Florianópolis, capital do Estado de Santa Catarina, Região Sul do Brasil. Neste período, a faixa etária do estudo correspondia a 249.530 pessoas, equivalente a $61,1 \%$ do total da população do município (IBGE - Instituto Brasileiro de Geografia e Estatística. Estimativa da população residente em 1o de Julho de 2009, de acordo com as cidades. http://www.ibge.gov.br, acessado em 15/Set/2009). O município apresentava o índice de desenvolvimento humano municipal (IDH-M) de 0,875 em 2000, situando-o na 4 a melhor posição dentre todos os municípios brasileiros 17 .

Esse estudo faz parte de um projeto maior denominado EpiFloripa (Estudo Epidemiológico das Condições de Saúde dos Adultos de Florianópolis, Santa Catarina), desenvolvido no âmbito do Programa de Pós-graduação em Saúde Coletiva, Universidade Federal de Santa Catarina (UFSC), em conjunto com pesquisadores do Programa de Pós-graduação em Nutrição e de Educação Física da UFSC. Vários desfechos e exposições foram investigados na pesquisa. Para o cálculo da amostra, utilizou-se a fórmula para o cálculo de prevalência, para uma amostra casual simples, considerando os seguintes parâmetros: prevalência de $50 \%$, erro amostral de 4 pontos percentuais e nível de $95 \%$ de confiança. O valor da amostra obtido foi de 599 adultos. Para o controle do efeito de delineamento do estudo, realizado em conglomerados, estimou um efeito de delineamento (deff) igual a 2. Foram ainda adicionados $10 \%$ para eventuais perdas e mais $20 \%$ para controle de fatores de confusão na análise multivariável, totalizando uma amostra igual a 1.581 adultos. Com o objetivo de aumentar o poder da amostra, ela foi definida em 2.016 adultos. Para esta pesquisa, considerou-se o tamanho da amostra obtido para o EpiFloripa adequado para detectar o risco relativo de pelo menos 1,3 , considerando uma prevalência de desfecho (artrite autorreferida) de $20 \%$ entre os não expostos, erro de $5 \%$ e poder de $80 \%$. Adicionalmente, a amostra final do EpiFloripa ( $\mathrm{n}=1.720$ ) foi suficiente para estimar a prevalência do desfecho artrite autorreferida ( $\mathrm{p}=7,7 \%$ ) com os seguintes parâmetros: erro amostral previsto de, no máximo, 2 pontos percentuais e nível de $95 \%$ de confiança (cálculos a posteriori).

O processo de amostragem foi realizado em conglomerados em dois estágios. As unidades do primeiro estágio foram os setores censitários, 
unidades de recenseamento do IBGE, constituídas de aproximadamente 300 domicílios cada. Os 420 setores urbanos de Florianópolis, de acordo com o Censo de 2000, foram estratificados em ordem crescente de renda, e foram sorteados sistematicamente 60 deles (com uma fração de amostragem igual a sete, selecionaram-se seis setores em cada decil em renda). As unidades de segundo estágio foram os domicílios. Uma etapa de arrolamento dos setores foi necessária para atualização dos setores, devido ao tempo decorrido desde o censo anterior à pesquisa, realizado em 2010. Para tanto, procedeu-se à contagem de todos os domicílios habitados de cada um dos setores censitários sorteados. Para definir a unidade amostral, os domicílios foram sorteados com uma fração de amostragem igual a oito, totalizando 2.094 domicílios sorteados. Foram excluídos da pesquisa adultos institucionalizados ou inaptos a responder ao questionário do estudo e mulheres grávidas. Não foram aceitos terceiros como respondentes. Considerou-se perdas os domicílios visitados por pelo menos três vezes sem que o entrevistador encontrasse a pessoa, incluindo-se uma visita em final de semana e outra noturna, ou caso houvesse recusa em participar.

A variável dependente foi estabelecida pela pergunta: "Algum médico ou profissional de saúde já disse que o (a) Sr.(a) tem artrite ou reumatismo?" conforme modelo de pergunta utilizado nos suplementos de saúde da PNAD e as respostas dicotomizadas em não e sim. As variáveis independentes foram: sexo, idade em anos completos (categorizada em 20-29; 30-39; 40-49 e 5059 anos), cor da pele autorreferida e categorizada segundo o IBGE (branca, parda, preta, amarela e indígena), escolaridade em anos de estudo completos (categorizada em 0-4; 5-8; 9-11 e 12 anos ou mais), renda familiar mensal per capita em reais, medida pelo total do ganho familiar dividido por todos os residentes no domicílio no mês anterior à visita (categorizada em tercis), índice de massa corporal ( $\mathrm{kg} / \mathrm{m}^{2}$ - IMC), de acordo com o peso e altura aferidos em uma balança digital da marca GA.MA Italy Professional, modelo HCM 5110M (Bolonha, Itália), com resolução de 100g e capacidade de $150 \mathrm{~kg}$, calibrada antes do início da pesquisa e por meio de um estadiômetro construído para o próprio estudo com uma fita métrica de resolução de $1 \mathrm{~mm} 18$ e categorizado segundo recomendação da OMS (baixo peso e normal $\leq 24,9 \mathrm{~kg} / \mathrm{m}^{2}$, sobrepeso $25-29,9 \mathrm{~kg} / \mathrm{m}^{2}$ e obesidade $\geq 30 \mathrm{~kg} / \mathrm{m}^{2}$ ), atividade física 19,20 (prática de algum tipo de exercício físico ou esporte nos últimos três meses), realização de consulta médica nas duas semanas anteriores à entrevista e o principal atendimento de saúde recebido (consulta médica de clínico geral, consulta de médico especialista, encaminhamento à emergência ou à internação hospitalar e somente marcação de consulta).

Os dados foram obtidos mediante entrevistas individuais realizadas nos domicílios sorteados por 35 entrevistadoras previamente treinadas em período integral durante cinco dias. Utilizou-se questionário padronizado e pré-codificado utilizando para armazenamento um computador de mão, personal digital assistants (PDA). Realizou-se estudo piloto em dois setores censitários que não fizeram parte da amostra. O controle de qualidade foi realizado em $20 \%$ dos pesquisados quando foram entrevistados pela segunda vez com uso de um questionário reduzido, aplicado pelo supervisor via telefone. Ainda, a comparação entre a faixa etária nas suas categorias e composição por sexo da população adulta de Florianópolis estimada pelo IBGE para 2009 foi comparada aos dados do projeto EpiFloripa.

$\mathrm{O}$ arquivo final foi exportado para o programa Stata 9.0 (Stata Corp., College Station, Estados Unidos). As variáveis de interesse foram analisadas quanto às suas distribuições. Para testar a associação entre artrite ou reumatismo com as variáveis independentes, utilizou-se o teste quiquadrado e, quando adequado, o teste de tendência linear de Wald. Em seguida, realizou-se a análise de regressão logística multivariável para verificar a associação dos fatores estudados com artrite ou reumatismo, estimando-se as razões de chance (OR) brutas e ajustadas e os respectivos intervalos de $95 \%$ de confiança. Para a entrada no modelo final multivariável, foram levadas em conta todas as variáveis que apresentaram o valor de $\mathrm{p}<0,20$ no teste do qui-quadrado. Permaneceram no modelo de regressão multivariável aquelas variáveis que mantiveram o valor de $\mathrm{p} \leq 0,05$ ou que ajustaram o modelo final. Para a inclusão das variáveis no modelo de regressão logística, os pesquisadores se basearam em um modelo teórico explicativo de determinação, conforme figura 21. Nessa relação, assumiu-se que os fatores mais distais determinam os do nível seguinte e assim subsequentemente até o desfecho. Sexo, idade e cor da pele autorreferida podem determinar a condição socioeconômica como a renda familiar mensal per capita em reais e a escolaridade dos indivíduos. Tais características podem influenciar as medidas antropométricas e o comportamento dos indivíduos como a realização de atividade física e o uso de serviços de saúde, contribuindo para o desfecho, a condição de saúde artrite ou reumatismo autorreferido.

Em todas as análises, considerou-se o efeito do desenho do estudo pelo uso do conjunto de comandos svy disponível no programa Stata e 


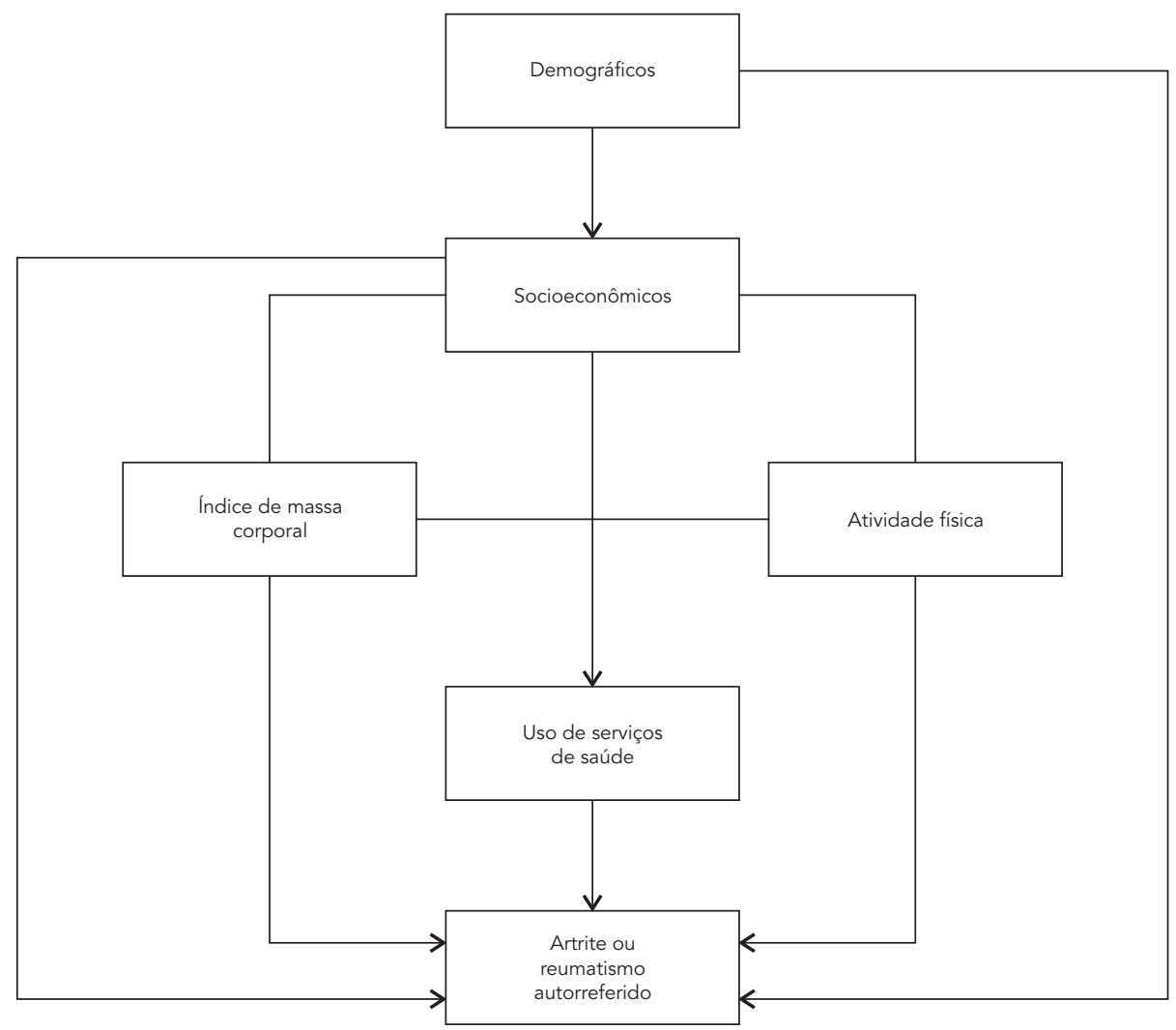

também pelos pesos dos indivíduos na amostra. Indivíduos que relataram a cor da pele amarela (1\%) e os indígenas $(1,2 \%)$, além daqueles que relataram encaminhamento para emergência ou internação hospitalar $(0,3 \%)$ e somente marcação de consulta $(0,1 \%)$ foram excluídos da análise logística em razão do baixo número encontrado na população de estudo.

A pesquisa foi submetida ao comitê de ética em pesquisa da UFSC (protocolo no. 351/08) e aprovada; todos os participantes assinaram o termo de consentimento livre e esclarecido.

\section{Resultados}

A taxa de resposta do estudo foi de $85,3 \%$ ( $\mathrm{n}=$ 1.720 pessoas). A confiabilidade foi considerada satisfatória com valor mínimo de kappa igual a 0,7 para, por exemplo, a variável atividade física, observando-se semelhança na distribuição das faixas etárias e composição do sexo entre a popu- lação adulta de Florianópolis estimada pelo IBGE para 2009 e a amostra do projeto EpiFloripa.

A maioria dos indivíduos era do sexo feminino $(55,8 \%)$, autorreferiu cor da pele branca $(84,2 \%)$, apresentou 12 ou mais anos de estudo (43\%), praticava atividade física nos últimos 3 meses $(51,5 \%)$ e apresentou IMC menor ou igual a $24,9 \mathrm{~kg} / \mathrm{m}^{2}(52,1 \%)$. Cerca de um terço $(31,4 \%)$ dos entrevistados tinha entre 20 e 29 anos de idade e a grande maioria das pessoas $(72,1 \%)$ não realizou atendimento médico nas duas semanas que antecederam à entrevista (Tabela 1). O IMC médio foi igual a $25,6 \mathrm{~kg} / \mathrm{m}^{2}$ (desvio-padrão = $4,9 \mathrm{~kg} / \mathrm{m}^{2}$ ) e a média de idade foi igual a 38 anos (desvio-padrão = 11,6 anos). A renda familiar mensal per capita revelou uma média de 1.433,04 reais (dados não apresentados).

A prevalência geral de artrite ou reumatismo autorreferido foi de 7,7\% (IC95\%: 6,4-8,9), sendo maior entre as mulheres $(10,1 \%)$. Quanto maior a idade, maior a prevalência de artrite autorreferida, o mesmo sendo observado em relação ao 
Descrição da amostra e da prevalência de artrite ou reumatismo autorreferido de acordo com as variáveis independentes em adultos. Florianópolis, Santa Catarina, Brasil, 2009.

\begin{tabular}{|c|c|c|c|c|}
\hline \multirow{2}{*}{$\begin{array}{l}\text { Variáveis } \\
\text { Total }\end{array}$} & \multicolumn{2}{|c|}{ Amostra } & \multirow{2}{*}{$\begin{array}{c}\begin{array}{c}\text { Artrite ou reumatismo } \\
\text { autorreferido } \\
\%(I C 95 \%)\end{array} \\
7,7(6,4-8,9)\end{array}$} & \multirow[t]{2}{*}{ Valor de $p$} \\
\hline & 1.720 & 100,0 & & \\
\hline $\operatorname{Sexo}(n=1.720)$ & & & & $<0,001$ \\
\hline Masculino & 761 & 44,2 & $4,7(3,3-6,1)$ & \\
\hline Feminino & 959 & 55,8 & $10,1(8,2-12,0)$ & \\
\hline Idade [anos] $(n=1.720)$ & & & & $<0,001 *$ \\
\hline $20-29$ & 540 & 31,4 & $2,7(1,4-4,1)$ & \\
\hline $30-39$ & 392 & 22,8 & $5,6(3,5-7,6)$ & \\
\hline $40-49$ & 438 & 25,5 & $8,4(5,9-11,0)$ & \\
\hline $50-59$ & 350 & 20,3 & $16,9(12,7-21,1)$ & \\
\hline Cor da pele $(n=1.715)$ & & & & 0,459 \\
\hline Branca & 1.444 & 84,2 & $8,0(6,6-9,3)$ & \\
\hline Parda & 147 & 8,5 & $6,1(1,8-10,3)$ & \\
\hline Preta & 87 & 5,1 & $6,8(0,9-12,8)$ & \\
\hline Amarela ** & 17 & 1,0 & - & \\
\hline Indígena ** & 20 & 1,2 & - & \\
\hline Escolaridade [anos completos] $(n=1.716)$ & & & & $<0,001$ * \\
\hline$\geq 12$ & 737 & 43,0 & $5,2(3,6-7,0)$ & \\
\hline $9-10$ & 568 & 33,1 & $7,0(4,8-9,2)$ & \\
\hline $5-8$ & 253 & 14,7 & $8,7(5,2-12,1)$ & \\
\hline$\leq 4$ & 158 & 9,2 & $19,7(13,6-25,8)$ & \\
\hline Renda familiar mensal per capita [Reais] $(n=1.685)$ & & & & 0,022 * \\
\hline Terceiro tercil (+ alto) & 559 & 33,2 & $6,4(4,3-8,5)$ & \\
\hline Segundo tercil & 562 & 33,3 & $6,9(4,8-9,0)$ & \\
\hline Primeiro tercil (+ baixo) & 564 & 33,5 & $10,1(7,4-12,8)$ & \\
\hline $\mathrm{IMC}\left[\mathrm{kg} / \mathrm{m}^{2}\right](\mathrm{n}=1.674)$ & & & & $<0,001$ * \\
\hline$\leq 24,9$ & 873 & 52,1 & $5,6(3,8-7,3)$ & \\
\hline $25-29,9$ & 531 & 31,8 & $8,6(6,3-11,0)$ & \\
\hline$\geq 30$ & 270 & 16,1 & $13,4(10,0-16,8)$ & \\
\hline Atividade física $(n=1.718)$ & & & & 0,038 \\
\hline $\operatorname{Sim}$ & 884 & 51,5 & $6,4(4,7-8,1)$ & \\
\hline Não & 834 & 48,5 & $9,1(7,2-11,0)$ & \\
\hline Consulta médica nas duas últimas semanas $(n=1.717)$ & & & & 0,018 \\
\hline Não realizou consulta & 1.236 & 72,0 & $6,8(5,4-8,1)$ & \\
\hline Realizou consulta & 481 & 28,0 & $10,1(7,0-13,2)$ & \\
\hline Principal atendimento médico recebido $(n=1.718)$ & & & & 0,058 \\
\hline Não realizou atendimento & 1.239 & 72,1 & $6,8(5,4-8,1)$ & \\
\hline \multicolumn{5}{|l|}{ Consulta de clínico geral } \\
\hline Consulta de especialista & 289 & 16,9 & $11,7(7,4-16,1)$ & \\
\hline Encaminhamento à emergência ou à internação hospitalar ** & 6 & 0,4 & - & \\
\hline Somente marcação de consulta ** & 5 & 0,3 & - & \\
\hline
\end{tabular}

IC95\%: intervalo de 95\% de confiança; IMC: índice de massa corporal.

* Teste de tendência linear de Wald;

** Excluídos da análise bivariada e multivariável. 
IMC. Associação inversa foi encontrada para renda e escolaridade, isto é, quanto menor a renda e a escolaridade, maior a prevalência do desfecho. Os indivíduos que referiam praticar atividade física nos últimos 3 meses apresentaram menor prevalência de artrite (Tabela 1).

A Tabela 2 mostra a análise bruta e ajustada entre o desfecho e as variáveis de interesse. Verifica-se na análise bruta que a chance do diagnóstico autorreferido de artrite entre as mulheres é maior do que entre os homens, e há um aumento da chance do desfecho à medida que a idade avança. Indivíduos com até 4 anos de estudo apresentaram maior chance da presença de diagnóstico de artrite autorreferido em comparação às pessoas com 12 anos ou mais. A chance da presença de artrite ou reumatismo foi maior entre os indivíduos com obesidade quando comparados aos indivíduos com IMC normal. Por fim, ter realizado consulta médica com especialista revelou maior associação com artrite ou reumatismo autorreferido. $\mathrm{Na}$ análise ajustada, as mulheres mantiveram mais que o dobro da chance de apresentar o desfecho e se manteve a mesma tendência de aumento na chance da presença do desfecho conforme o aumento da idade. Presença de artrite ou reumatismo manteve uma associação inversa com escolaridade, observando que indivíduos com 4 anos ou menos de estudos tiveram quase três vezes maior chance de apresentar o desfecho quando comparados aos mais escolarizados. A associação entre IMC e o desfecho foi parcialmente confundida, mas manteve a associação no modelo ajustado, identificando que entre indivíduos obesos a chance do desfecho foi $70 \%$ maior do que entre aqueles com IMC menor do que $25 \mathrm{~kg} / \mathrm{m}^{2}$. Ter consultado com especialista nas duas semanas anteriores à entrevista apresentou-se no limite da significância estatística para maior chance de associação com o desfecho quando comparado com aqueles que não realizaram atendimento médico após ajuste pelas outras variáveis.

\section{Discussão}

A pesquisa identificou que existem diferenças no diagnóstico de artrite autorreferida segundo características socioeconômicas e demográficas. Indivíduos do sexo feminino, com mais de 30 anos de idade, aqueles menos escolarizados e obesos apresentaram uma chance maior de artrite ou reumatismo autorreferido.

A prevalência de artrite ou reumatismo, segundo a PNAD 2008 e o inquérito de saúde realizado em Belo Horizonte, Minas Gerais, no ano de 2003, mostrou prevalências de $5,7 \%$ na po- pulação adulta 10,22 , menor do que a observada na nossa pesquisa $(7,7 \%)$. Vale ressaltar que há diferenças metodológicas entre os três estudos. Por exemplo, o limite inferior da faixa etária da população adulta na PNAD foi de 18 anos. Além disso, a forma como os dados foram obtidos também é distinta. Na PNAD, 50,9\% das respostas foram obtidas por outro informante e no inquérito de Belo Horizonte o mesmo ocorreu em 29 \% dos casos. Por outro lado, no projeto EpiFloripa não foram aceitos terceiros como respondentes.

A prevalência de artrite ou reumatismo observada em Florianópolis foi menor do que a estimada nos Estados Unidos no ano de 2010 $(29,6 \%)$, conforme projeções realizadas para faixa etária a partir dos 20 anos ${ }^{5}$. Essa projeção foi realizada com base nos dados da Behavioral Risk Factor Surveillance Survey (BRFSS) de 2003, inquérito de saúde realizado via telefone com a população norte-americana, sobre fatores de risco comportamentais para doenças crônicas. $\mathrm{O}$ achado de uma prevalência maior de artrite ou reumatismo entre as mulheres corrobora os achados apresentados na literatura. A oscilação e o perfil dos níveis de hormônios femininos 1 , com destaque para a menopausa, e a maior utilização dos serviços de saúde pelas mulheres 11,23, consequentemente aumentando a chance de diagnóstico, pode contribuir para explicar essa diferença.

A tendência de aumento do diagnóstico de artrite com o aumento da idade observada no nosso estudo é corroborada por outras pesquisas como, por exemplo, o inquérito mundial de saúde realizado no Brasil em 2003 13. De forma similar, observa-se o aumento na prevalência de artrite autorreferida a partir dos 30 anos no Brasil 10.

Os estudos internacionais demonstram uma associação entre cor da pele e presença de artrite ou reumatismo, apontando que indivíduos de cor da pele branca seguida das de cor preta 24 apresentaram uma chance maior do desfecho. Contudo, estudo longitudinal norte-americano evidenciou que, embora a prevalência autorreferida dessa condição de saúde seja maior entre os brancos, isso se justifica pelo maior acesso ao atendimento médico. Por outro lado, os brancos apresentam menor incidência no desenvolvimento de incapacidade, devido à melhor condição social e menor presença de comorbidades entre indivíduos desse grupo 25 . Nesta pesquisa não se identificou associação entre o desfecho e cor da pele.

A prevalência de artrite autorreferida foi inversamente proporcional ao nível de escolaridade, corroborando os achados da PNAD 2003, na qual os indivíduos com até 4 anos de estudo 
Análise de regressão logística bruta e ajustada entre artrite ou reumatismo autorreferido e as variáveis independentes em adultos. Florianópolis, Santa Catarina, Brasil, 2009.

\begin{tabular}{|c|c|c|c|c|}
\hline \multirow[t]{2}{*}{ Variáveis } & \multicolumn{2}{|c|}{ Análise bruta } & \multicolumn{2}{|c|}{ Análise ajustada } \\
\hline & OR (IC95\%) & Valor de $\mathrm{p}$ & OR (IC95\%) & Valor de $p$ \\
\hline Sexo & & $<0,001$ & & $<0,001$ \\
\hline Masculino & 1,0 & & 1,0 & \\
\hline Feminino & $2,2(1,5-3,2)$ & & $2,2(1,5-3,2)$ & \\
\hline Idade (anos) & & $<0,001$ & & $<0,001$ \\
\hline 20-29 & 1,0 & & 1,0 & \\
\hline $30-39$ & $2,0(1,1-3,7)$ & & $2,0(1,1-3,6)$ & \\
\hline $40-49$ & $3,2(1,8-5,6)$ & & $3,0(1,7-5,4)$ & \\
\hline $50-59$ & $7,1(4,0-12,5)$ & & $6,9(3,9-12,2)$ & \\
\hline Cor da pele & & 0,423 * & & 0,734 \\
\hline Preta/Parda & 1,0 & & 1,0 & \\
\hline Branca & $1,2(0,7-2,2)$ & & $1,1(0,6-1,9)$ & \\
\hline Escolaridade em anos completos & & $<0,001$ & & 0,001 \\
\hline$\geq 12$ & 1,0 & & 1,0 & \\
\hline $9-11$ & $1,3(0,8-2,1)$ & & $1,3(0,8-2,1)$ & \\
\hline $5-8$ & $1,7(0,9-2,9)$ & & $1,4(0,8-2,4)$ & \\
\hline$\leq 4$ & $4,4(2,5-7,6)$ & & $2,8(1,6-5,0)$ & \\
\hline Renda familiar mensal per capita (reais) & & 0,046 & & 0,255 ** \\
\hline Terceiro tercil (+ alta) & 1,0 & & 1,0 & \\
\hline Segundo tercil & $1,0(0,6-1,7)$ & & $1,0(0,5-1,8)$ & \\
\hline Primeiro tercil (+ baixa) & $1,6(1,0-2,6)$ & & $1,3(0,7-2,5)$ & \\
\hline $\mathrm{IMC}\left(\mathrm{kg} / \mathrm{m}^{2}\right)$ & & $<0,001$ & & 0,022 \\
\hline$\leq 24,9$ & 1,0 & & 1,0 & \\
\hline $25-29,9$ & $1,5(0,9-2,5)$ & & $1,1(0,6-1,8)$ & \\
\hline$\geq 30$ & $2,6(1,7-3,9)$ & & $1,7(1,1-2,7)$ & \\
\hline Atividade física & & 0,041 & & 0,868 ** \\
\hline Sim & 1,0 & & 1,0 & \\
\hline Não & $1,4(1,0-2,0)$ & & $1,0(0,7-1,5)$ & \\
\hline $\begin{array}{l}\text { Consulta médica nas duas últimas } \\
\text { semanas }\end{array}$ & & 0,032 & & 0,203 ** \\
\hline Não realizou consulta & 1,0 & & 1,0 & \\
\hline Realizou consulta & $1,5(1,0-2,3)$ & & $1,3(0,8-2,0)$ & \\
\hline Principal atendimento médico recebido & & 0,014 & & 0,073 \\
\hline Não realizou consulta & 1,0 & & 1,0 & \\
\hline Consulta de clínico geral & $1,2(0,6-2,2)$ & & $0,9(0,5-1,8)$ & \\
\hline Consulta de especialista & $1,8(1,1-2,9)$ & & $1,6(1,0-2,6)$ & \\
\hline
\end{tabular}

IC95\%: intervalo de 95\% de confiança; IMC: índice de massa corporal; OR: odds ratio.

* Não incluída na análise multivariável (valor $p>0,20)$;

** Excluídos do modelo hierárquico final (valor de $p>0,20$ ).

Nota: amostra do modelo final: $n=1.651$.

tiveram mais diagnóstico de artrite quando comparados aos indivíduos com 12 anos ou mais 26 . Semelhante constatação pode estar relacionada a piores condições de saúde, traduzida pela dificuldade de acesso a atendimento de qualidade $\mathrm{e}$ desequilíbrio das condições socioeconômicas no
Brasil. Situação parecida foi encontrada quanto à renda, o mesmo sendo detectado num estudo australiano realizado com adultos que demonstrou que artrite é desproporcionalmente maior em indivíduos com baixa renda ${ }^{8}$. As PNAD dos anos de 1998 e 2003 apresentaram, após análise 
ajustada, as OR iguais a 1,61 e 1,18, respectivamente, quando comparados o quintil de renda mais baixo com o quintil de renda mais alto 27 . Todavia, a influência da renda no presente estudo sobre a presença de artrite dependeu do nível de escolaridade dos indivíduos.

O IMC apresenta uma associação direta e proporcional com artrite ou reumatismo, o mesmo sendo observado em outros estudos 16,28. Quanto maior o IMC, maior a chance dessa condição, além de ser um fator independente entre homens e mulheres. Um inquérito de saúde norteamericano realizado no ano de 2005 indicou que as pessoas obesas foram 1,9 vez mais propensas a relatar artrite em comparação com os entrevistados de peso normal ${ }^{28}$, evidenciando a associação entre artrite e obesidade como resultante do estresse mecânico sobre a articulação ${ }^{2}$.

A atividade física ausente ou insuficiente apresenta uma relação direta com a artrite. O grupo de estudo do Colégio Americano de Reumatologia (American College of Rheumatology - ACR) recomendou algum nível de atividade física para a manutenção da saúde das articulações tanto normais quanto artríticas. Apesar disso, a maioria dos adultos nos Estados Unidos não realiza atividade física suficiente para obter benefícios para a saúde articular 29. No presente estudo, a presença de atividade física perdeu a associação com o desfecho quando controlada pelos fatores mais distais como renda e escolaridade.

Algumas limitações devem ser consideradas nesta pesquisa. O desenho transversal do estudo impossibilita a determinação de causa e efeito entre as variáveis exploratórias e o desfecho. Com base nos resultados obtidos, ressalta-se a possibilidade de causalidade reversa característica nos estudos de delineamento transversal. Por exemplo, os indivíduos que autorrelataram ter artrite ou reumatismo podem ter realizado mais consultas médicas do que os livres dessa condição por causa da presença do agravo. Outro fator a se levar em conta, diz respeito ao modo de coleta do desfecho que pode limitar a comparação com outros estudos. Utilizou-se a mesma pergunta da PNAD para facilitar a comparação com os estudos brasileiros 10,11,13. Não obstante, existe a possibilidade do viés de memória na coleta de algumas informações, atenuado pela aferição do peso e da altura para o cálculo do IMC, pela limitação do período recordatório de três meses para prática de atividade física e de um mês para renda familiar, além da característica comum da artrite ou reumatismo ser um agravo crônico. Por último, a limitação deste estudo, que utilizou dados de autorrelato da condição reumática, pode ocorrer em razão da não confirmação diagnóstica por um médico. Por outro lado, vários inquéritos de saúde revelam que as informações obtidas sobre a prevalência de doenças crônicas apresentam boa concordância quando comparadas aos registros médicos ou exames clínicos, especialmente para algumas patologias crônicas 30,31. No que se refere à artrite ou reumatismo, um estudo realizado para determinar a validade e a confiabilidade entre o diagnóstico autorreferido de artrite e a avaliação médica reumatológica (padrão-ouro) mostrou boa sensibilidade (70,3\%) e especificidade $(72,4 \%) 32$.

A prevalência da artrite autorreferida depende do acesso e uso ao serviço de saúde. As pessoas com maior acesso ao atendimento médico têm mais chance de receber o diagnóstico. $\mathrm{O}$ resultado deste estudo mostra que a artrite ou reumatismo não está associado à consulta com clínico geral ou especialista mesmo após análise ajustada.

O monitoramento da prevalência dos fatores de risco para doenças crônicas não transmissíveis (DCNT) é uma das ações mais importantes para programar medidas preventivas de maior poder custo-efetivo. Essa conduta se torna mais evidente quando consideramos os agravos de natureza comportamental, entre outras artrite ou reumatismo. As DCNT são de etiologia multifatorial e compartilham vários fatores de riscos que podem ser modificados. Os resultados deste estudo permitem concluir que artrite ou reumatismo apresenta considerável prevalência na população adulta em relação ao cenário nacional mais recente. Ainda, foi possível identificar fatores associados não modificáveis como o sexo e a idade e também fator passível de modificação tal como a escolaridade e a obesidade, este último considerado fator de risco comum a diferentes agravos crônicos. A urgência em deter o crescimento das DCNT no país justifica a adoção de estratégias integradas e sustentáveis de prevenção e controle dessas doenças, assentadas sobre seus principais fatores de risco modificáveis.

Inquéritos têm demonstrado repetidamente que entre $40 \%$ a $60 \%$ dos indivíduos com artrite ou reumatismo autorreferido não estão recebendo tratamento ${ }^{33}$. Políticas públicas de saúde poderiam incorporar programas específicos de prevenção a fatores relacionados à artrite ou reumatismo autorreferido. A obesidade e o sedentarismo poderiam ser prevenidos pela manutenção do peso corporal adequado e estímulo à prática de atividade física regular, ambas sob supervisão de uma equipe multidisciplinar de saúde contribuindo, dessa forma, para diminuição da prevalência e do impacto da artrite tanto para o indivíduo quanto para a sociedade, pelo enfrentamento dos fatores de risco comuns a diferentes doenças. Acesso aos serviços de saúde 
na atenção primária para possibilitar diagnóstico precoce e, quando oportuno, na atenção secundária com médico especialista, complementariam a estratégia para as políticas públicas. Sugerem-se novas pesquisas de base populacional com o objetivo de aumentar a consistência das informações sobre prevalência de artrite e para investigar fatores associados em outras regiões do Brasil.

\section{Resumo}

Estimar a prevalência de artrite ou reumatismo autorreferido e os fatores associados. Realizou-se um estudo transversal de base populacional em Florianópolis, Santa Catarina, Brasil, com 1.720 adultos entre 20 e 59 anos. A presença de artrite ou reumatismo autorreferido foi analisada por meio do modelo hierárquico de determinação no nível demográfico, socioeconômico, comportamental e uso de serviços de saúde. Utilizouse análise de regressão logística para avaliar a associação entre as variáveis. A prevalência de artrite ou reumatismo autorreferido foi de 7,7\% (IC95\%: 6,4-8,9). A chance de artrite ou reumatismo autorreferido fo $i$ duas vezes maior entre as mulheres, maior entre aqueles com índice de massa corporal (IMC) $\geq 30 \mathrm{~kg} / \mathrm{m}^{2}$, diretamente proporcional à idade e inversamente proporcional à escolaridade. A prevalência de artrite ou reumatismo autorreferido foi maior do que a estimativa nacional no ano de 2008. Essa realidade sugere a necessidade de um planejamento de políticas públicas voltado para esse agravo de saúde.

Artrite; Doenças Reumáticas; Adulto; Prevalência

\section{Colaboradores}

R. S. Gomes foi responsável pelo planejamento do estudo, análise e interpretação dos dados e a redação do artigo. K. G. Peres orientou o planejamento do projeto, análise e interpretação dos dados, a redação do artigo, conduziu a revisão crítica do conteúdo, além de dar a aprovação final à versão a ser publicada.

\section{Agradecimentos}

Aos técnicos do Instituto Brasileiro de Geografia e Estatística (IBGE) pelo suporte na fase de treinamento do estudo; à professora doutora Nilza Nunes da Silva, do Departamento de Epidemiologia, Faculdade de Saúde Pública, Universidade de São Paulo, pelas contribuições com os procedimentos de amostragem; à Secretaria Municipal de Saúde de Florianópolis pelo auxílio na operacionalização da pesquisa; e aos discentes dos Programas de Pós-Graduação em Saúde Coletiva, Educação Física e Nutrição, Universidade Federal de Santa Catarina, que realizaram a função de supervisores do estudo. Ao Conselho Nacional de Desenvolvimento Científico e Tecnológico (CNPq), sob nº. 485327/2007-4 - Edital Universal 2007 que foi a fonte financiadora do Projeto EpiFloripa 2009 - Estudo Epidemiológico das Condições de Saúde dos Adultos de Florianópolis, Santa Catarina. 


\section{Referências}

1. Silva VRL, Menezes AMB, Noal RB. Sintomas articulares crônicos em adultos de Pelotas, Rio Grande do Sul, Brasil: prevalência e determinantes. Cad Saúde Pública 2009; 25:2571-82.

2. Wilkins K. Incident arthritis in relation to excess weight. Health Rep 2004; 15:39-49.

3. Picavet HS, Hazes JM. Prevalence of self reported musculoskeletal diseases is high. Ann Rheum Dis 2003; 62:644-50.

4. Machado GM, Barreto SM, Passos VM, Lima-Costa MF. Health status indicators among communitydwelling elders with arthritis: the Bambui health and aging study. J Rheumatol 2006; 33:342-7.

5. Fontaine KR, Haaz S, Heo M. Projected prevalence of US adults with self-reported doctor-diagnosed arthritis, 2005 to 2050. Clin Rheumatol 2007; 26:772-4.

6. Centers for Disease Control and Prevention. Racial/ethnic differences in the prevalence and impact of doctor-diagnosed arthritis - United States, 2002. MMWR Morb Mortal Wkly Rep 2005; 54: 119-23.

7. National Institute on Disability and Rehabilitation Research. Notice of proposed Long-range plan for fiscal years 1999-2004. Department of Education Federal Register 1998; 63:57189-219.

8. Busija L, Hollingsworth B, Buchbinder R, Osborne $\mathrm{RH}$. Role of age, sex, and obesity in the higher prevalence of arthritis among lower socioeconomic groups: a population-based survey. Arthritis Rheum 2007; 57:553-61.

9. Badley EM, Tennant A. Impact of disablement due to rheumatic disorders in a British population: estimates of severity and prevalence from the Calderdale Rheumatic Disablement Survey. Ann Rheum Dis 1993; 52:6-13.

10. Barros MBA, Francisco PMSB, Zanchetta LM, César CLG. Tendências das desigualdades sociais e demográficas na prevalência de doenças crônicas no Brasil, PNAD: 2003- 2008. Ciênc Saúde Coletiva 2011; 16:3755-68.

11. Pinheiro RS, Viacava F, Travassos C, Brito AS. Gênero, morbidade, acesso e utilização de serviços de saúde no Brasil. Ciênc Saúde Coletiva 2002; 7:687-707.

12. Lima-Costa MF, Barreto SM, Giatti L. Condições de saúde, capacidade funcional, uso de serviços de saúde e gastos com medicamentos da população idosa brasileira: um estudo descritivo baseado na Pesquisa Nacional por Amostra de Domicílios. Cad Saúde Pública 2003; 19:735-43.

13. Theme-Filha MM, Szwarcwald CL, Souza-Junior PRB. Socio-demographic characteristics, treatment coverage, and self-rated health of individuals who reported six chronic diseases in Brazil, 2003. Cad Saúde Pública 2005; 21 Suppl 1:S43-53.

14. Souza DCC, Minto CM, Ciconelli RM. Atendimento em reumatologia no Município de São Paulo: análise na gestão plena do Sistema Único de Saúde (SUS)-Ano 2004. Rev Bras Reumatol 2006; 46: 93-102.
15. Secretaria de Estado da Saúde do Distrito Federal. Reumatologia - da atenção básica à atenção terciária - programa de atenção integral aos pacientes com doenças do tecido conjuntivo ou acometimento do sistema músculo-esquelético, 2008. http:// www.saude.df.gov.br/sites/300/311/00000688.pdf (acessado em 12/Mai/2011).

16. Mili F, Helmick CG, Zack MM. Prevalence of arthritis: analysis of data from the US Behavioral Risk Factor Surveillance System, 1996-99. J Rheumatol 2002; 29:1981-8.

17. Programa das Nações Unidas para o Desenvolvimento. Atlas do desenvolvimento humano no Brasil. Brasília: Programa das Nações Unidas para o Desenvolvimento; 2003.

18. Lohman TG, Roche AF, Martorell R. Anthropometric standardization reference manual. Champaign: Human Kinetics Books; 1988.

19. Secretaria de Vigilância em Saúde, Ministério da Saúde. VIGITEL Brasil 2008. Vigilância de fatores de risco e proteção para doenças crônicas por inquérito telefônico. Brasília: Ministério da Saúde; 2009.

20. Sousa TF, Nahas MV, Silva DAS, Duca GFD, Peres MA. Fatores associados à obesidade central em adultos de Florianópolis, Santa Catarina: estudo de base populacional. Rev Bras Epidemiol 2011; 14:296-309.

21. Victora CG, Huttly SR, Fuchs SC, Olinto MT. The role of conceptual frameworks in epidemiological analysis: a hierarchical approach. Int J Epidemiol 1997; 26:224-7.

22. Lima-Costa MF. A saúde dos adultos na Região Metropolitana de Belo Horizonte: um estudo epidemiológico de base populacional. Belo Horizonte: Núcleo de Estudos em Saúde Pública e Envelhecimento Fundação Oswaldo Cruz/Universidade Federal de Minas Gerais; 2004.

23. Vidal RQS, Neto AMS. Trabalhadoras brasileiras: características socioeconômicas e ocupacionais e perfil de saúde, Brasil, 2003. Rev Bras Saúde Ocup 2009; 34:115-27.

24. Centers for Disease Control and Prevention. Prevalence of doctor: diagnosed arthritis and arthritisattributable activity limitation - United States, 2007-2009. MMWR Morb Mortal Wkly Rep 2010; 59:1261-5.

25. Song J, Chang HJ, Tirodkar M, Chang RW, Manheim LM, Dunlop DD. Racial/ethnic differences in ADL disability among older adults with arthritis: a longitudinal study. Arthritis Rheum 2007; 57: 1058-66.

26. Barros MBA, César CLG, Carandina L, Torre GD. Desigualdades sociais na prevalência de doenças crônicas no Brasil, PNAD-2003. Ciênc Saúde Coletiva 2006; 11:911-26.

27. Lima MFC, Matos DL, Camarano AA. Evolução das desigualdades sociais em saúde entre idosos e adultos brasileiros: um estudo baseado na pesquisa nacional por amostra de domicílios (PNAD 1998, 2003). Ciênc Saúde Coletiva 2006; 11:941-50. 
28. Zakkak JM, Wilson DB, Lanier JO. The association between body mass index and arthritis among US adults: CDC's surveillance case definition. Prev Chronic Dis 2009; 6:A56.

29. Fontaine KR, Heo M, Bathon J. Are US adults with arthritis meeting public health recommendations for physical activity? Arthritis Rheum 2004; 50:624-8.

30. Martin CM, Leffe N, Calonge N, Garrett C, Nelson DE. Validity of self-reported chronic conditions and health services in a managed care population. Am J Prev Med 2000; 18:215-8.
31. Haapanen N, Miilunpalo S, Pasanen M, Oja P, Vuori I. Agreement between questionnaire data and medical records of chronic diseases in middle-aged and elderly Finnish men and women. Am J Epidemiol 1997; 145:762-9.

32. Bombard JM, Powell KE, Martin LM, Helmick CG, Wilson WH. Validity and reliability of self-reported arthritis: Georgia Senior Centers, 2000-2001. Am J Prev Med 2005; 28:251-8.

33. Badley EM, Kasman NM. The impact of arthritis on Canadian women. BMC Womens Health 2004; 4 Suppl 1:S18.

Recebido em 08/Dez/2011

Versão final reapresentada em 15/Abr/2012

Aprovado em 03/Mai/2012 